\section{A miniaturized ultrawideband Archimedean spiral antenna for low-power sensor applications in energy harvesting}

\author{
Antonio Alex-Amor | Pablo Padilla | \\ José M. Fernández-González । \\ Manuel Sierra-Castañer
}

KEYWORDS

Archimedean spiral antenna, energy harvesting, low-power applications, radiofrequency, ultrawideband

\section{1 | INTRODUCTION}

Radiofrequency (RF) energy harvesting is based on the use of the available power at certain frequencies of the electromagnetic spectrum for device power supply. The constant increase in the number of RF transmitters has led to a nonnegligible level of power at some particular frequency bands. This fact, joined to the reduction in the power consumption necessities of many devices, has turned RF energy harvesting into a feasible energy source for such lowconsumption devices, being an alternative to other wellestablished energy harvesting techniques, as those related with solar, kinetic or wind energy, ${ }^{1}$ especially when considering ultralow-power sensor applications. For instance, as discussed in earlier study, ${ }^{2}$ several harvesting devices are starting to be used for medical purposes, in order to measure intraocular pressure, temperature, electrocardiograms (ECG), etc.; or in fields as wireless sensor networks (WSN). Most of these devices consume less than $30 \mu \mathrm{W}$.

Generally, a RF energy harvesting system has four subsystems $^{3}$ : an antenna that acquires the available environmental energy; a rectifier circuit that provides a DC supply; a matching circuit, placed between the antenna and the rectifier circuit, that maximizes the delivered power to the circuit; and a storage circuit that retains the conditioned power. Regarding the harvesting element, it was already discussed in the study ${ }^{4}$ that wideband antennas, such as those shown $\mathrm{in}^{5,6}$, are preferable instead of multiband antennas. They are most times easier to design, and they are interoperable among countries, whose frequency assignment plans can be different among them. In these terms, spiral antennas arise as a proper option. They also provide omnidirectional patterns, high efficiency, and most of them are circularly polarized, which is of great interest in order to unnecessarily avoid neglecting part of the available energy, as the direction of arrival and polarization of the incoming signals are still unknown.

Finally, it is of paramount importance to guarantee that the antenna covers DTT (400 MHz), LTE (800, 2100, $2600 \mathrm{MHz})$, GSM $(900,1800 \mathrm{MHz}), \mathrm{WiFi}(2400,5200 \mathrm{MHz})$, and 
WiMAX $(3500 \mathrm{MHz})$ bands, ${ }^{7}$ since almost all relevant RF power sources are situated at those frequency ranges.

\section{I ANTENNA DESIGN AND SIMULATION}

When choosing which type of spiral to use, factors such as the purity of the circular polarization or the bandwidth of the spiral should be taken into account. In the study of El-Feshawy, ${ }^{8}$ a comparison between equiangular and Archimedean spiral antennas is made. It is pointed out that the Archimedean spiral shows an improved axial ratio and a wider bandwidth for a given outer diameter. The design and manufacturing of the Archimedes spiral is also easier than in the case of the equiangular spiral, due to the constant angular increase of its arms. Besides that, wideband spiral antennas may be configured with single, double, or multiple arms, ${ }^{9}$ the multiple-arm spiral antenna being a good option to provide squinted beams. In the light of previous works, ${ }^{8,9}$ the two-arm Archimedean spiral antenna seems as the proper option to be implemented in practice.

The Archimedean spiral is typically classified within the group of frequency independent antennas, that is, antennas that maintain some of their radiation parameters constant, as the input impedance or the bandwidth, in relation to the frequency. Victor H. Rumsey laid the foundations of this subject. According to him, ${ }^{10}$ those antennas that are entirely defined by angles, as the Archimedean spiral or the equiangular spiral, show a frequency-independent behavior. Theoretically, an infinitesized Archimedean spiral antenna in a self-complementary design (the arm width $w$ is equal to the gap width $s$ ) presents a constant impedance that is fixed to the value $Z_{\text {ant }}=Z_{\text {comp }}=60$ $\pi \Omega \approx 188.5 \Omega$, where $Z_{\text {comp }}$ is the impedance of its

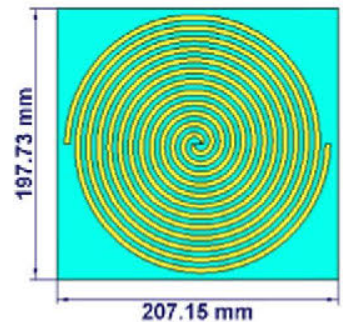

(A)

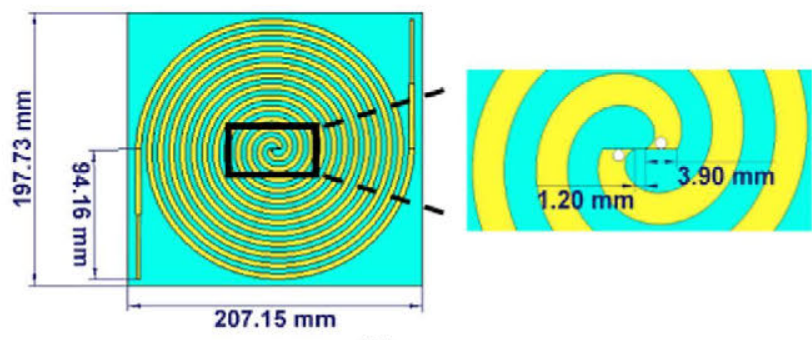

(C)

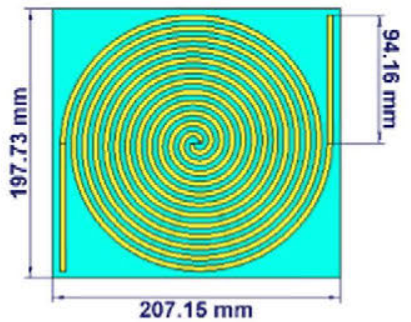

(B)
FIGURE 1 Different designs of the Archimedean spiral antenna: (A) no miniaturization ${ }^{4}$, (B) miniaturization without impedance step, and (C) miniaturization with an impedance step [Color figure can be viewed at wileyonlinelibrary.com] complement. The finite size of an Archimedes antenna causes a finite bandwidth, which becomes more reduced as the antenna becomes smaller, with its impedance varying nearby $188.5 \Omega$. This fact can be noticed in Figure 6 .

On the other hand, several studies emphasize $e^{3,4,11,12}$ that the current distribution over the surface of the Archimedean spiral is concentrated close to the center of the antenna at high frequencies and opens to its end at low frequencies. This current distribution leads to the equations presented, ${ }^{4}$ where the dimensions of the antenna are estimated according to the desired bandwidth, determined by the lower and the upper cutoff frequencies, $\mathrm{f}_{\mathrm{L}}$ and $\mathrm{f}_{\mathrm{H}}$, respectively. Due to the complex geometry of the antenna, this dimension estimation is revealed as a good starting point to model the Archimedes spiral on a full-wave electromagnetic simulator.

\section{1 | Miniaturization}

A design of a circularly polarized Archimedean spiral (Figure 1(A)) was already presented by us, ${ }^{4}$ the antenna showing a bandwidth range from $0.51 \mathrm{GHz}$ to $18 \mathrm{GHz}$. In order to increase the RF harvested power per unit area $\left(\mu \mathrm{W} / \mathrm{cm}^{2}\right)$, it is essential to try to reduce the physical dimensions of the antenna. Several miniaturization techniques have been studied so far, most of them based on structural modifications and lumped element loadings. ${ }^{13-15}$. Recent studies have tried to reduce the size of spiral antennas by using fractal geometries, as in ${ }^{16}$, where a modified Koch curve is used to shrink a Fibonacci spiral antenna (FSA). We propose increasing the bandwidth of the Archimedean spiral by extending the arms to the end of the antenna (maintaining the same PCB area). Consequently, the electrical size of the Archimedes antenna is higher and therefore, it reaches lower frequencies without significant performance degradation. Figure 1 depicts different designs following that approach.

As the simulation results probe (Figure 2), it is preferable to place an impedance step (Figure 1(C)) when extending the arms of the antenna, so that reflections are softened and

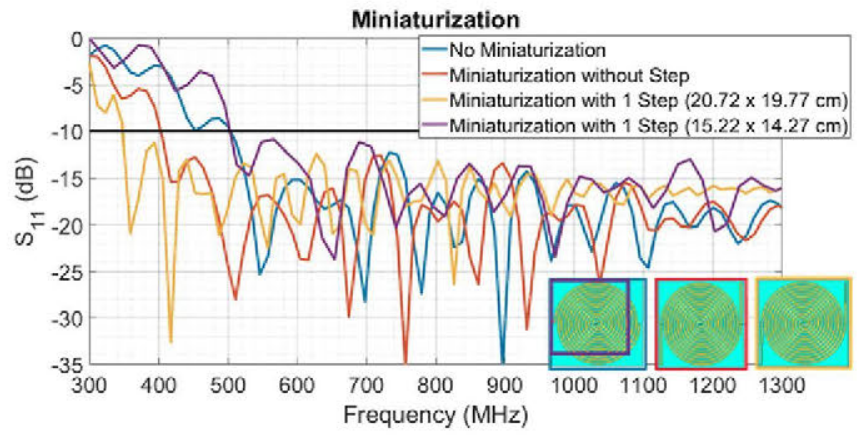

FIGURE 2 Simulated reflection coefficient of the Archimedean spiral antenna (normalized to $188.5 \Omega$ ) with different designs: (A) no miniaturization ${ }^{4}$, (B) miniaturization without impedance step, and (C) miniaturization with an impedance step $(20.72 \times 19.77 \mathrm{~cm}$ vs $15.22 \times 14.27 \mathrm{~cm})$ [Color figure can be viewed at wileyonlinelibrary.com] 


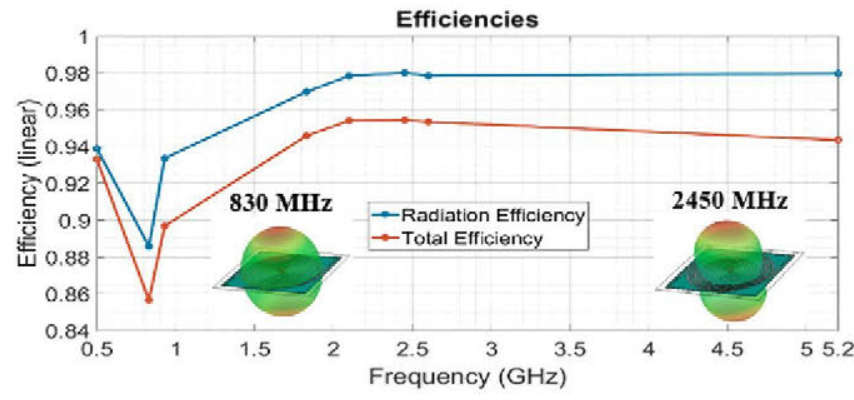

FIGURE 3 Simulated efficiencies (radiation efficiency and total efficiency) of the miniaturized (one impedance step) Archimedean spiral antenna, and two examples of the spiral's 3D radiation pattern at two different frequencies of interest [Color figure can be viewed at wileyonlinelibrary.com]

more bandwidth is achieved $\left(\mathrm{f}_{\mathrm{L} 1 \text {-step }}=350 \mathrm{MHz}\right.$ vs $\mathrm{f}_{\text {L0-step }}=400 \mathrm{MHz}$ vs $\mathrm{f}_{\text {Lno } \min }=510 \mathrm{MHz}$ ). In these terms, Figure 2 (purple line) shows that the area of the miniaturized antenna can be reduced $5.5 \times 5.5 \mathrm{~cm}^{2}$ to meet the lower cutoff frequency $(510 \mathrm{MHz})$ of the nonminiaturized antenna.

In Figure 6 (blue line), it is presented the reflection coefficient of the miniaturized spiral (with an impedance step). Whether we set the reference on $-10 \mathrm{~dB}$, the antenna covers from $0.35 \mathrm{GHz}$ to $16 \mathrm{GHz}$. In Figure 3 , the radiation efficiency and the total efficiency of the miniaturized Archimedes antenna are plotted. It shows a good efficiency behavior, with values over the $86 \%$ at the frequencies of interest. Besides that, Figure 4 presents the axial ratio of the antenna, at the different frequencies of interest, in two cuts in the planes $\phi=0^{\circ}$ and $\phi=90^{\circ}$. As it can be seen, the spiral is circularly polarized in a wide range of elevation angles $\left(-65^{\circ}\right.$ to $\left.65^{\circ}\right)$ at most frequencies, but $500 \mathrm{MHz}$, where it could not go under $3 \mathrm{~dB}$ and remains elliptically polarized.

\section{I ANTENNA PROTOTYPE AND MEASUREMENT RESULTS}

Figure 5 presents a prototype of the miniaturized Archimedean spiral antenna. The antenna of the picture was

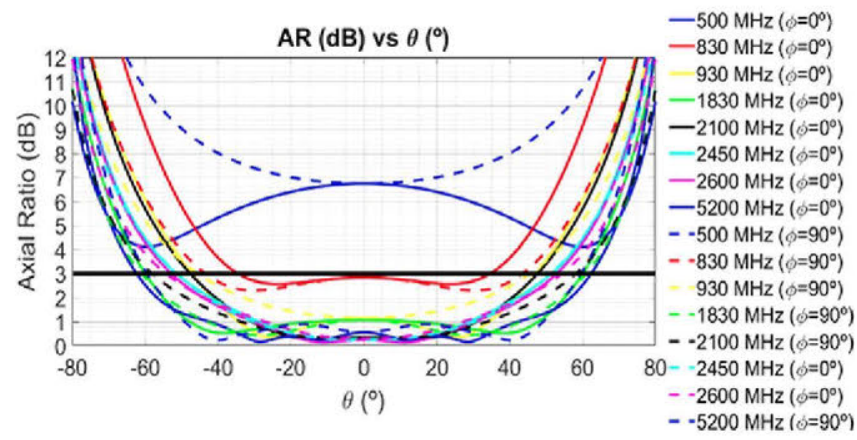

FIGURE 4 Simulated axial ratio, at different frequencies, of the Archimedean spiral antenna in two cuts in the planes $\phi=0^{\circ}$ (continuous line) and $\phi=90^{\circ}$ (dashed line) [Color figure can be viewed at wileyonlinelibrary.com]

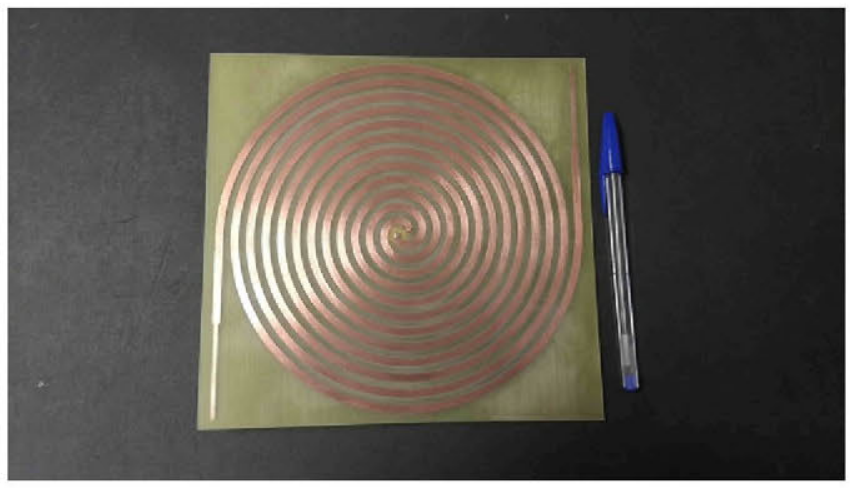

FIGURE 5 Prototype of the miniaturized Archimedean spiral antenna [Color figure can be viewed at wileyonlinelibrary.com]

constructed making use of a LPKF ProtoMat S100 milling machine $^{17}$ on a low-cost FR4 substrate $\left(\varepsilon_{\mathrm{r}}=4.7, \tan \delta=\right.$ 0.014 @ $1 \mathrm{MHz}$ ). As previously mentioned, the dimensions of the antenna are $19.77 \mathrm{~cm} \times 20.72 \mathrm{~cm}$.

\section{1 | Reflection coefficient measurement}

We characterize in the laboratory the performance of the Archimedean spiral antenna in two measurement steps. In the first of them, we extract the reflection coefficient of the antenna from an Agilent 8722ES network analyzer, in order to check its bandwidth. Subsequently, the power spectrum is acquired with a N9020A MXA signal analyzer in two different scenarios: inside and outside the laboratory.

In Figure 6, it is shown a comparison between the simulated and measured reflection coefficients of the Archimedean spiral. As the experimental results probe (red line in Figure 6), the antenna is well-matched $(<-10 \mathrm{~dB})$ from $350 \mathrm{MHz}$ to $16 \mathrm{GHz}$, presenting an ultrawideband behavior. Besides that, its return loss is superior to $8.5 \mathrm{~dB}$ in the entire frequency range shown $(0.3-20 \mathrm{GHz})$. On the other hand, although the measured reflection coefficient is slightly higher than the simulated one, note how the lower and upper cutoff frequencies coincide almost perfectly, being their values $350 \mathrm{MHz}$ and $16 \mathrm{GHz}$, respectively.

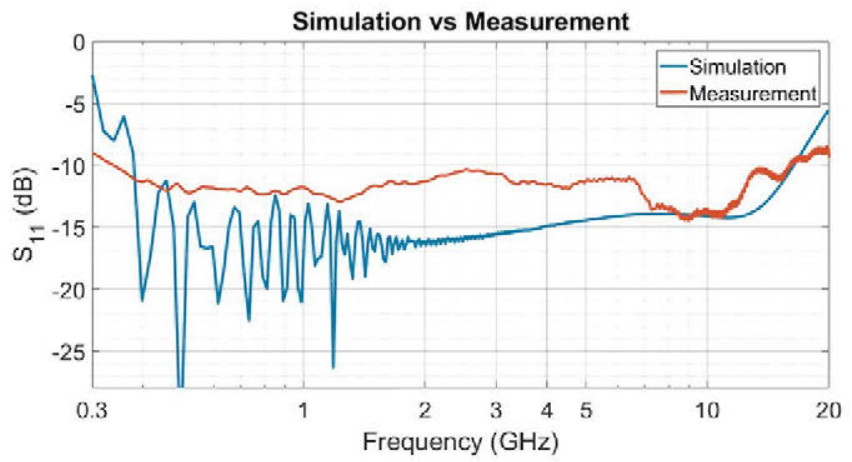

FIGURE 6 Comparison of the simulated and measured reflection coefficients of the Archimedean spiral antenna (normalized to $188.5 \Omega$ ) [Color figure can be viewed at wileyonlinelibrary.com] 


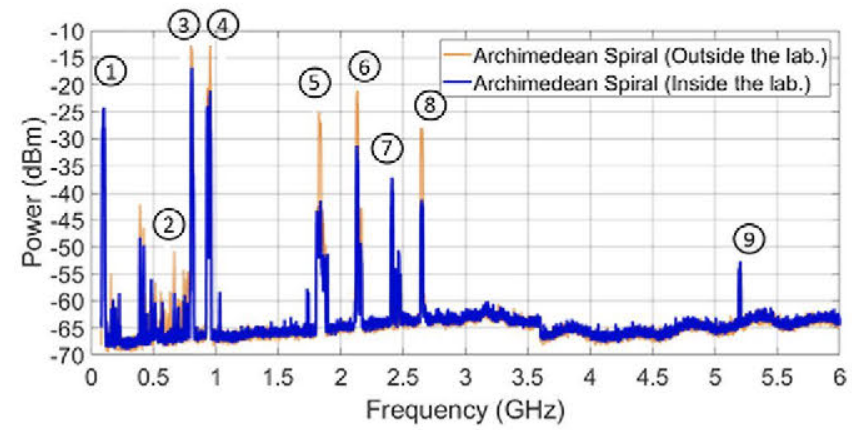

FIGURE 7 Power spectrum measured by the Archimedean spiral antenna inside and outside the laboratory with the most relevant bands remarked: 1-FM, 2-DTT, 3-LTE-800, 4-GSM-900, 5-GSM1800, 6-LTE-2100, 7-WiFi, 8-LTE-2600, 9-WiFi [Color figure can be viewed at wileyonlinelibrary.com]

\section{2 | Power spectrum measurement. Harvested power}

In order to obtain a realistic measurement of the harvested power, the antenna is connected to a signal analyzer capable of integrating the power spectrum over a prefixed bandwidth. Figure 7 shows the power spectrum acquired by the miniaturized Archimedean spiral antenna in two different scenarios: inside and outside the laboratory (our laboratory is located inside the Higher Technical School of Telecommunication Engineers, Technical University of Madrid, and the nearest base station [LTE-800, GSM-900, GSM-1800] is situated at a distance of $60 \mathrm{~m}$ and at a height of $8 \mathrm{~m}$ from the receiving Archimedean antenna). As it can be noticed in Figure 7, there is no significant contribution of power in any of them from $5200 \mathrm{MHz}$ onwards, where the $-65 \mathrm{dBm}$ noise level prevails. From this frequency value, only militar and satcom applications can be found, but the directivity of the antenna is so reduced that it does not allow us to acquire anything remarkable. Despite capturing noise in a large frequency range $(5.2 \mathrm{GHz}-16 \mathrm{GHz})$, its contribution to the total power harvested is negligible; hence, it is not worthy to try to raise the upper cutoff frequency of the antenna (although, in this particular case, it is something intrinsic to its inner radius and it does not hinder the design).

As seen in Figure 7, the measured power level of the spectrum peaks is generally higher outside the laboratory than inside the laboratory, due to the radio signal attenuation caused by the building itself (position of the room, walls, windows, etc. ${ }^{19}$ ). Most of the energy captured comes from cellular bands, reaching almost $-10 \mathrm{dBm}$ for the 830 and $930 \mathrm{MHz}$ bands. However, the measured FM spectral peak is higher than expected ( $-25 \mathrm{dBm}$ outside the laboratory), even though the antenna is not specially designed to cover FM band. On the other hand, power contribution of WiFi bands is surprisingly smaller than expected, leaving them in the background.

It could be of interest comparing the power harvested by the Archimedean spiral antenna with other type of baseline antennas, such as dipoles or microstrip patches. In these

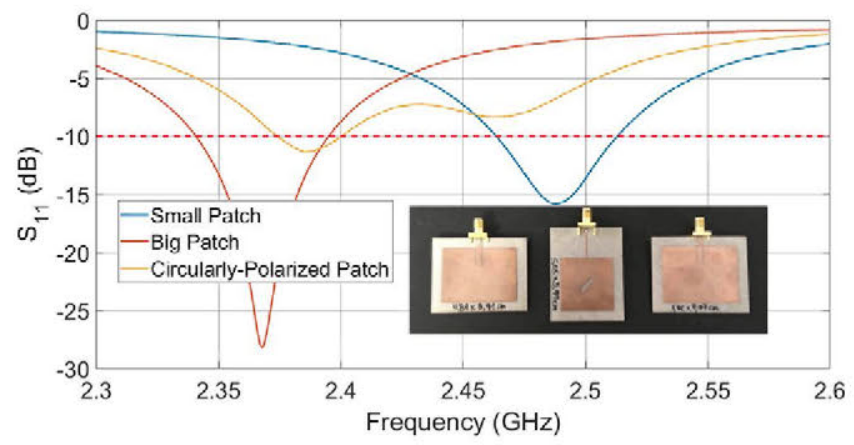

FIGURE 8 Measured reflection coefficient of the manufactured 2.4-GHz patches (dimensions: Small patch: $4.89 \times 3.91 \mathrm{~cm}$, circularly-polarized patch: $5.05 \times 3.91 \mathrm{~cm}$, big patch: $5.02 \mathrm{x}$ $4.07 \mathrm{~cm}$ ) [Color figure can be viewed at wileyonlinelibrary.com]

terms, three different patches that operate within the $2.4 \mathrm{GHz}$ band were designed and manufactured, as shown in Figure 8 . One of them (the one in the center of the image that is matched with a $\lambda / 4$ transformer) is circularly polarized due to the slot inserted in the center of the patch, while the other two are linearly polarized. Figure 9 shows a comparison between the power spectrum measured by the Archimedean spiral antenna (inside the laboratory) and by the three $2.4 \mathrm{GHz}$ patches. The limited bandwidth of the microstrip patches, even within the WiFi band, naturally conditions the measured spectrum peaks to be smaller than in the case of using the Archimedean spiral antenna.

The total harvested power, which is the available power between the arms of the Archimedean spiral antenna, is calculated as the sum of all carriers present in the radio spectrum. The result of integrating the power spectrum shown in Figures 7 and 9 leads to the values presented in Table 1 . We should remark that all measurements have been carried out in a realistic scenario, that is, where no directional sources have been intentionally put into scene. As previously commented, the power acquired outside the laboratory $(1.86 \mathrm{dBm})$ is higher than the one acquired inside the laboratory $(-3.19 \mathrm{dBm})$, both of which are sufficient to provide

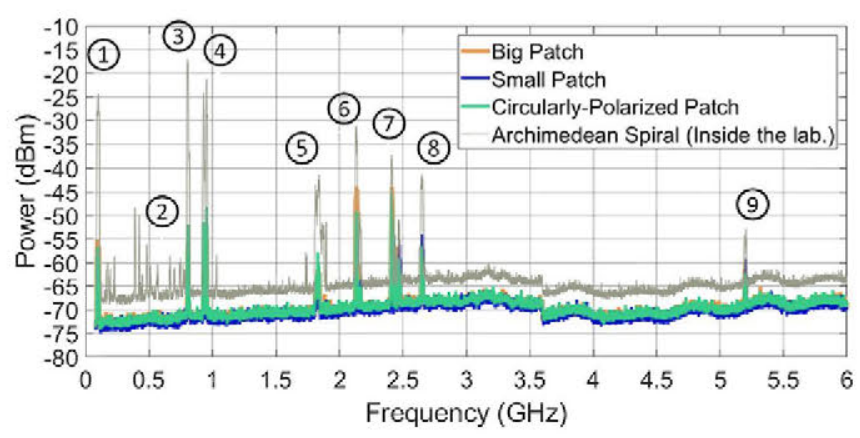

FIGURE 9 Comparison between the power spectrum measured by the Archimedean spiral antenna inside the laboratory and by the three 2.4-GHz patches, with the most relevant bands remarked: 1-FM, 2-DTT, 3-LTE-800, 4-GSM-900, 5-GSM-1800, 6-LTE2100, 7-WiFi, 8-LTE-2600, 9-WiFi [Color figure can be viewed at wileyonlinelibrary.com] 
TABLE 1 Measurements of the harvested power

\begin{tabular}{|c|c|c|c|c|}
\hline & \multicolumn{2}{|c|}{ INSIDE THE LAB. } & \multicolumn{2}{|c|}{ OUTSIDE THE LAB } \\
\hline & Power (dBm) & Power/area $\left(\mu \mathrm{W} / \mathrm{cm}^{2}\right)$ & Power (dBm) & Power/area $\left(\mu \mathrm{W} / \mathrm{cm}^{2}\right)$ \\
\hline Archimedean spiral $(0.3-16 \mathrm{GHz})$ & -3.19 & 1.17 & 1.86 & 3.75 \\
\hline Small patch $(2.4 \mathrm{GHz})$ & -28.68 & 0.071 & - & - \\
\hline Circularly-polarized patch $(2.4 \mathrm{GHz})$ & -26.69 & 0.11 & - & - \\
\hline Horn $(0.8-18 \mathrm{GHz})^{18}$ & -10 (max.) & - & - & - \\
\hline
\end{tabular}

the amount of power required $(1-30 \mu \mathrm{W})$ by the elements discussed in Section I.

The best way to compare the amount of harvested power among antennas with different sizes is to express the acquired power per area unit, that is, the physical dimensions of the Archimedes spiral are $19.77 \mathrm{~cm} \times 20.72 \mathrm{~cm}$, which leads to $3.75 \mu \mathrm{W} / \mathrm{cm}^{2}$ and $1.17 \mu \mathrm{W} / \mathrm{cm}^{2}$ outside and inside the laboratory, respectively. In Table 1, it is also presented the total power harvested by the three microstrip patches and an ultrawideband horn used in the study of Sun et al. ${ }^{18}$ Note that despite the fact that the patch area is much smaller than the spiral area, the harvested power per area unit inside the laboratory is about 10 times lower than in the case of the Archimedes spiral. On the other hand, it is also mentioned in the table that some measurements in Sun et al study ${ }^{19}$ show that a power level of $-10 \mathrm{dBm}$ was acquired by a broadband horn, the nearest cell station being situated in this case at $150 \mathrm{~m}$.

\section{4 | CONCLUSIONS}

This paper presents the design, simulation, manufacturing, and measurement of a high-efficiency circularly polarized ultrawideband Archimedean spiral antenna. Due to its frequency-independent behavior, the antenna is capable of operating from $0.3 \mathrm{GHz}$ to $16 \mathrm{GHz}$, as the experimental results probe. A few miniaturization techniques were also essayed on the antenna, being demonstrated that by extending the arms of the spiral, we increase $160 \mathrm{MHz}$ its bandwidth in the lower frequencies, which is equivalent to reduce $5.5 \times 5.5 \mathrm{~cm}^{2}$ the area of the miniaturized antenna. Finally, two tests were carried out with the antenna, inside and outside the laboratory, in order to estimate the incoming power level. These results lead to the conclusion that the major contribution of the harvested power comes from the cellular bands, especially from 800 and $900 \mathrm{MHz}$.

\section{ACKNOWLEDGMENT}

This work has been supported by the project ENABLING5G, "Enabling Innovative Radio Technologies for 5G networks" (TEC2014-55735-C3-1-R) of the Ministerio de Economía, Industria y Competitividad. Antonio Alex-Amor and Pablo Padilla also acknowledge partial support from the project TIN2016-75097-P of the Spanish Ministerio de Economía, Industria y Competitividad.

The authors would like to thank Jose Luis Masa-Campos, Pablo Sánchez-Olivares and Eduardo García-Marín, all belonging to Universidad Autónoma de Madrid, for the use of their LPKF ProtoMat S100 milling machine on the construction of the Archimedean spiral antenna; and Alfonso T. Muriel-Barrado for his help in certain measurement steps. All simulations have been carried out using CST-MS with a cooperation agreement between CST and Universidad Politécnica de Madrid (UPM).

\section{ORCID}

Antonio Alex-Amor (D) https://orcid.org/0000-0003-0776-1315

\section{REFERENCES}

[1] Mateu L, Moll F. Review of energy harvesting techniques and applications for microelectronics. Proc SPIE - Int Soc Opt Eng 5837. 2005.

[2] Tran L, Cha H, Park W. RF power harvesting: a review on designing methodologies and applications. Micro Nano Syst Lett. 2017; 5:14.

[3] Hagerty JA, Helmbrecht FB, McCalpin WH, Zane R, Popovic ZB. Recycling ambient microwave energy with broad-band Rectenna arrays. IEEE Trans Microw Theory Techn. 2004;52(3):1014-1024.

[4] Alex-Amor A, Fernández-González JM, Padilla P, Sierra-Castañer M. Comparison Between a Multiband PIFA and an Ultrawideband Archimedean Spiral Antenna for Energy Harvesting in Microwave Bands. 2nd URSI AT-RASC, 2018. Manuscript accepted for publication.

[5] Lee C, Park J, Lee J. Wideband tapered monopole antenna with 2 by 2 resonant loop array for electromagnetic energy harvesting and microwave power transmission. Microw Opt Technol Lett. 2017;59(4):797-802.

[6] Simón J, Flores-Gonzalez JR, González-Salas JS, Ordaz-Salazar FC, Flores-Troncoso J. A log-periodic toothed trapezoidal antenna for RF energy harvesting. Microw Opt Technol Lett. 2015;57(12):2765-2768.

[7] Ministerio de Energía, Turismo y Agenda Digital, Gobierno de España. "Registro Público de Concesiones". Retrieved March 19, 2018, from https://sedeaplicaciones.minetur.gob.es/setsi regconcesiones/

[8] El-Feshawy MH, Hammad H. Comparison between broadband equiangular and Archimedean spiral rectennas for energy harvesting. 33rd National Radio Science Conference (NRSC). 2016. 
[9] Afsar MN, Wang Y, Cheung R. Analysis and measurement of a broadband spiral antenna. IEEE Antennas Propag Mag. 2004; 46(1):59-64 .

[10] Rumsey VH. Frequency Independent Antennas. IRE International Convention Record. 1957;5:114-118.

[11] Balanis CA. Antenna Theory: Analysis and Design. 3rd ed. Hoboken, New Jersey: John Wiley \& Sons; 2005.

[12] Caswell ED. Design and Analysis of Star Spiral with Application to Wideband Arrays with Variable Element Sizes (Doctoral Thesis). Bradley Department of Electrical and Computer Engineering, Virginia Polytechnic Institute and State University

[13] Mehrabani A, Shafai L. Polarisation reconfigurable, centre-fed, and low-profile Archimedean spiral antennas with unidirectional broadside patterns. IET Microw Antennas Propag. 2016.

[14] Volakis JL, Chen C, Fujimoto K. Small Antennas: Miniaturization Techniques \& Applications. 1st ed. Pennsylvania Plaza New York City: McGraw Hill Education; 1987.

[15] Liu Q, Ruan CL, Peng L, Wu WX. A novel compact Archimedean spiral antenna with gap-loading. Prog Electromagn Res Lett. 2008;3:169-177.
[16] Sharma C, Vishwakarma DK. Miniaturization of spiral antenna based on Fibonacci sequence using modified Koch curve. Antennas Wirel Propag Lett. 2017;16:932-935.

[17] LPKF Laser \& Electronics. Manual ProtoMat S100. http://www. lpkfusa.com/downloads/support/docs/man_s100.pdf

[18] Sun H, Guo Y, He M, Zhang Z. A dual-band Rectenna using broadband Yagi antenna Array for ambient RF power harvesting. Antennas Wirel Propag Lett. 2013;12:918-921.

[19] Wells PI. The attenuation of UHF radio signals by houses. IEEE Trans Vehicular Technol. 1977;VT-26(4):358-362. 